\title{
Opposite metabolic responses of shoots and roots to drought
}

CHEMICAL BIOLOGY

CLIMATE SCIENCES

Received

10 June 2014

Accepted

3 October 2014

Published

29 October 2014

Correspondence and requests for materials should be addressed to A.G.-G. la.gargallo@ creaf.uab.es)
Albert Gargallo-Garriga ${ }^{1,2,3}$, Jordi Sardans ${ }^{1,2}$, Míriam Pérez-Trujillo ${ }^{3}$, Albert Rivas-Ubach ${ }^{1,2}$, Michal Oravec ${ }^{4}$, Kristyna Vecerova ${ }^{4}$, Otmar Urban ${ }^{4}$, Anke Jentsch $^{5}$, Juergen Kreyling ${ }^{6}$, Carl Beierkuhnlein ${ }^{6}$, Teodor Parella ${ }^{3}$ \& Josep Peñuelas ${ }^{1,2}$

${ }^{1}$ CSIC, Global Ecology Unit CREAF-CEBAB-CSIC-UAB, Cerdanyola del vallès, 08193, Catalonia, Spain, ${ }^{2}$ CREAF, Cerdanyola del vallès, 08193, Catalonia, Spain, ${ }^{3}$ Service of Nuclear Magnetic Resonance and Department of Chemistry, Faculty of Sciences and Biosciences, Universitat Autònoma de Barcelona, Bellaterra, 08913, Catalonia, Spain, ${ }^{4}$ Global Change Research Centre, Academy of Sciences of the Czech Republic, Belidla 4a, CZ-60300 Brno, Czech Republic, ${ }^{5}$ Disturbance Ecology and Vegetation Dynamics, University of Bayreuth, D95440 Bayreuth, Germany, ${ }^{6}$ Department of Biogeography, University of Bayreuth, D-95440 Bayreuth, Germany.

Shoots and roots are autotrophic and heterotrophic organs of plants with different physiological functions. Do they have different metabolomes? Do their metabolisms respond differently to environmental changes such as drought? We used metabolomics and elemental analyses to answer these questions. First, we show that shoots and roots have different metabolomes and nutrient and elemental stoichiometries. Second, we show that the shoot metabolome is much more variable among species and seasons than is the root metabolome. Third, we show that the metabolic response of shoots to drought contrasts with that of roots; shoots decrease their growth metabolism (lower concentrations of sugars, amino acids, nucleosides, N, P, and $\mathrm{K}$ ), and roots increase it in a mirrored response. Shoots are metabolically deactivated during drought to reduce the consumption of water and nutrients, whereas roots are metabolically activated to enhance the uptake of water and nutrients, together buffering the effects of drought, at least at the short term.

P lants have complex and intricate regulatory machinery that coordinates the demands of physiological activity, growth, and development. Plants regulate their shoot/root ratios of biomass in response to the availability of substrates and to environmental changes ${ }^{1}$. Shoots and roots have different functions: shoots essentially have a photosynthetic function, whereas roots take up water and nutrients. Shoots and roots may thus compete for the resources that a plant acquires ${ }^{2,3}$. Plants under different resource availabilities differentially allocate the available resources to shoots and roots to optimise the efficiency of their use ${ }^{4-7}$. Plants generally allocate relatively fewer resources to their roots when light is low and the availabilities of water and nutrients are high, consistent with the resource optimisation hypothesis ${ }^{8}$. For example, the up-regulation of root growth under reduced supplies of nitrogen was confirmed by a meta-analysis of published data'. Moreover, differential allocation to root and leaves has been observed as a response to biotic factors under various competitive condition ${ }^{10}$ or to various soil physicochemical traits ${ }^{11}$. Several models based on carbon balance have been developed to explain the mechanisms behind the shoot/root allocation of carbon ${ }^{12-14}$. Plants, however, are likely to respond to perturbations in the growth environment not only by altering their allocation of biomass to shoots and roots, but also by changing the metabolic activities of these organs. We hypothesised that shoots and roots would present contrasting metabolisms in response to changing environmental conditions given their different physiological functions and their different roles in the acclimation to stress. We subjected two common grass species (Holcus lanatus L. and Alopecurus pratensis L.) to drought conditions in the field (a simulated 1000-year recurrence of drought in a long-term experiment of rainfall manipulation in a semi-natural grassland at Bayreuth, Germany) and used metabolomics to test this hypothesis. We thus analysed metabolomes, which can be defined as the totality of thousands of compounds of low molecular weight (metabolites) required for maintenance, growth, and cellular functions of an organism at a given time. We also conducted elemental analyses to simultaneously assess the metabolic and stoichiometric responses of shoots and roots to drought ${ }^{15-17}$.

The responses of plants to drought are crucial because drought is one of the most important environmental stressors for plants, and changes in the shoot/root ratios of biomass have been frequently observed in response to drought ${ }^{18,19}$. Moreover, the frequency and length of droughts are projected to increase under global climate change ${ }^{20,21}$, and more regions are expected to be affected by severe droughts ${ }^{21-24}$. We have previously reported a significant impact of drought on the metabolomes of plant species at the foliar level and have observed that these 
changes are strongly linked to changes in the elemental C/N/P/K stoichiometry ${ }^{25}$. The simultaneous analysis of the metabolism and the nutrient stoichiometries of both shoots and roots that we now conducted assessed the allocation of metabolites and nutrients for various plant functions, such as growth, defence, reproduction, mechanisms of health, and avoidance of stress at the level of whole plants, thereby assessing the likely contrasting responses of shoots and roots to experimental drought and to seasonally changing conditions.

In addition to the expected differences between the two species and between the two seasons of sampling, July and September, we observed clear differences between shoots and roots at both the metabolic and elemental concentration levels (Fig. 1). Principal component analyses (PCAs) of both metabolomic and stoichiometric data showed that different species and seasons had different scores along the second PC axis (Fig. 1), but the most significant changes in metabolomic structure were between shoots and roots, which were separated along the first PC axis (Fig. 1). These results were confirmed by PERMANOVA analysis (species: pseudo- $F=51.2, P<$ 0.001 ; season: pseudo- $F=43.4, P<0.001$; shoot/root: pseudo- $F=$ 154.4, $P<0.001)$. Shoots had higher concentrations of nucleotides, compounds related to the metabolism of amino acids and sugars, osmolytes, and secondary metabolites such as terpenes and phenols, while the roots had higher concentrations of amino acids and sugars irrespective of season and species (Fig. 2). The concentrations of C, $\mathrm{N}, \mathrm{P}$, and $\mathrm{K}$ and $\mathrm{C} / \mathrm{P}$ ratio were higher in shoots than in roots, whereas the $\mathrm{C} / \mathrm{N}, \mathrm{C} / \mathrm{K}, \mathrm{N} / \mathrm{K}, \mathrm{P} / \mathrm{K}$, and $\mathrm{N} / \mathrm{P}$ ratios and the concentration of Fe were lower (Table S1). Roots had lower concentrations of nitrogenous bases and higher concentrations of most soluble sugars than did shoots (Figs. 2 and 3), likely due to the need of energy for the assimilation of soil resources, such as nutrients, and for root growth ${ }^{26,27}$. Most of the compounds that participated in these functions, such as compounds related to the metabolism of amino acids and sugars, should be synthesised in leaves but allocated and used in large amounts in roots.

The observed variability of the metabolome was lower in the root samples than in the shoot samples (Fig. 1). The shifts in the metabolome of roots among different individual plants, species, and seasons were much less significant and smaller than those of the shoots. The coefficient of variation of the PC2 scores was 15\% for roots and $57 \%$ for shoots. The metabolism of roots thus appears to be much more conservative and homeostatic than that of shoots.

Our results notably showed that the metabolome and stoichiometry of shoots and roots of both plant species responded to drought in opposite ways (Figs. 4 and 5). The results of the PCA were confirmed by the PERMANOVA analysis (treatment $\times$ shoot/ root interaction; pseudo- $F=3.0 ; P<0.001$ ). The concentrations of choline and glycine betaine, which are involved in osmotic protec$\operatorname{tion}^{28}$, and of gamma-aminobutyric acid and primary metabolites decreased in the shoots, and terpenes and metabolites related to anti-stress mechanisms increased in shoots under drought (Figs. 4A). Contrasting changes were observed in the metabolome of roots and shoots for both species and for both seasons (Figs. S1 and S2 and Tabs. S1-S4). The concentration of gamma-aminobutyric acid increased in roots, suggesting that roots remain more active than shoots under drought stress ${ }^{29}$. Moreover, the concentrations of N, P, and $\mathrm{K}$ decreased in roots but increased in shoots (Fig. 2). The concentrations of primary metabolites thus increased in roots. A decrease in primary metabolism combined with an increase in some secondary metabolites in shoots in response to drought is consistent with the decrease in the shoot/root ratio usually observed under drought ${ }^{30,31}$. The results are also consistent with the hypothesis that plants allocate more carbon to antistress mechanisms under drought $^{32}$, and with the previous results in this experimental site: reduced leaf water potential, leaf gas exchange, leaf protein content and efficiency of photosynthetic light conversion, and increased leaf carbon isotope signature and leaf carbohydrate content in response to drought ${ }^{33}$. Terpenes were the secondary metabolites that presented highest responsiveness to drought (Fig. S3 and tab. S1-S4). The function of terpenes are still controversial, but they seem to protect the plants against abiotic and biotic stresses ${ }^{34}$.

The results provide clear evidence that plants have a high capacity to modulate and vary the allocation of nutrients and the relative activities of different metabolic pathways for producing biomass in both shoots and roots. Primary metabolites in plants, such as sugars, amino acids, and fatty acids, are synthesised mainly in the photosynthetic tissues of shoots, where the concentrations of these metabolites are higher than in roots. Under drought conditions, however, the plasticity of the plants allow a shift to increased synthesis or allocation of several primary metabolites to roots while decreasing allocation to shoots. These results have important implications for ecological studies. First, they provide clear evidence of the complexity of the stoichiometric shifts in terrestrial plants in response to environmental gradients and changes. The N/P ratio decreases in roots under drought conditions, coinciding with the investment in root growth, whereas this ratio increases in shoots, which is consistent with the growth rate hypothesi ${ }^{35}$. The results, however, also indicate that the study of N/P ratios only in the leaves of terrestrial plants is unable to provide a general view of the relationships of N/P ratios with ecological traits and gradients. Second, the shift in the allocation of metabolites and nutrients from shoots to roots in response to drought demonstrates the high capacity of plant metabolomes to respond modularly to stressors. When water is a limiting factor, metabolites involved with energy production and growth (especially sugars and amino acids) are shifted from shoots to roots. The simultaneous ecometabolomic analysis of roots and shoots can provide a complete view of the entire plant, including the response of different organs to environmental changes, the global phenotypic response, and the metabolic mechanisms underlying these responses. Such a simultaneous analysis has shown that shoots and roots have different metabolomes and nutrient concentrations, the shoot metabolome is much more variable than the root metabolome, and roots and shoots respond to drought with opposite metabolic changes. When metabolism is activated in roots, metabolism is deactivated in shoots. These opposite metabolic responses may account for the frequent lack of large reductions in productivity in drought experiments, at least for short term ${ }^{36}$.

\section{Methods}

Field experiment and sampling. Instant metabolomic and stoichiometric responses to a simulated 1000-year recurrence of drought (complete exclusion of rainfall for 42 days prior to the first sampling) and its subsequent effects to the end of the growing season three months later, and after irrigation with the deficit amount of rainfall, were assessed for the common C3 grasses A. pratensis and H. lanatus in a long-term experiment of rainfall manipulation in a semi-natural grassland at Bayreuth, Germany (EVENT II experiment ${ }^{37}$ ). Samples from the cases $(2$ species $\times 2$ organs (leaf blades and fine roots) $\times 2$ sampling dates $\times 3$ precipitation manipulations $\times 15$ plots) were collected in the field, immediately frozen, and further prepared as in Rivas-Ubach et al. $(2013)^{38}$. See the supplementary material for details.

Liquid chromatography-mass spectrometry (LC-MS) analysis. LC-MS chromatograms were obtained using a Dionex Ultimate 3000 HPLC system (Thermo Fisher Scientific/Dionex RSLC, Dionex, Waltham, Massachusetts, USA) coupled to an LTQ Orbitrap XL high-resolution mass spectrometer (Thermo Fisher Scientific, Waltham, Massachusetts, USA) equipped with an HESI II (heated electrospray ionisation) source. Chromatography was performed on a reversed-phase C18 Hypersil gold column $(150 \times 2.1 \mathrm{~mm}, 3-\mu$ particle size; Thermo Scientific, Waltham, Massachusetts, USA) at $30^{\circ} \mathrm{C}$. The mobile phases consisted of water ( $0.1 \%$ acetic acid) (A) and acetonitrile (B).

Nuclear magnetic resonance (NMR) analysis. All NMR experiments were performed using a Bruker AVANCE 600 spectrometer equipped with an automatic sample changer and a multinuclear triple resonance TBI probe (BrukerBiospin, Rheinstetten, Germany) at a field strength of $14.1 \mathrm{~T}\left(600.13 \mathrm{MHz}{ }^{1} \mathrm{H}\right.$ frequency) and at $298.0 \mathrm{~K}$. Following the introduction to the probe, the samples were allowed to equilibrate $(2 \mathrm{~min})$ prior to shimming to ensure good homogeneity of the magnetic field. All spectra were referenced to trimethylsilyl propionate (TSP) $\left({ }^{1} \mathrm{H}\right.$ and ${ }^{13} \mathrm{C}$ at $\delta$ 
A
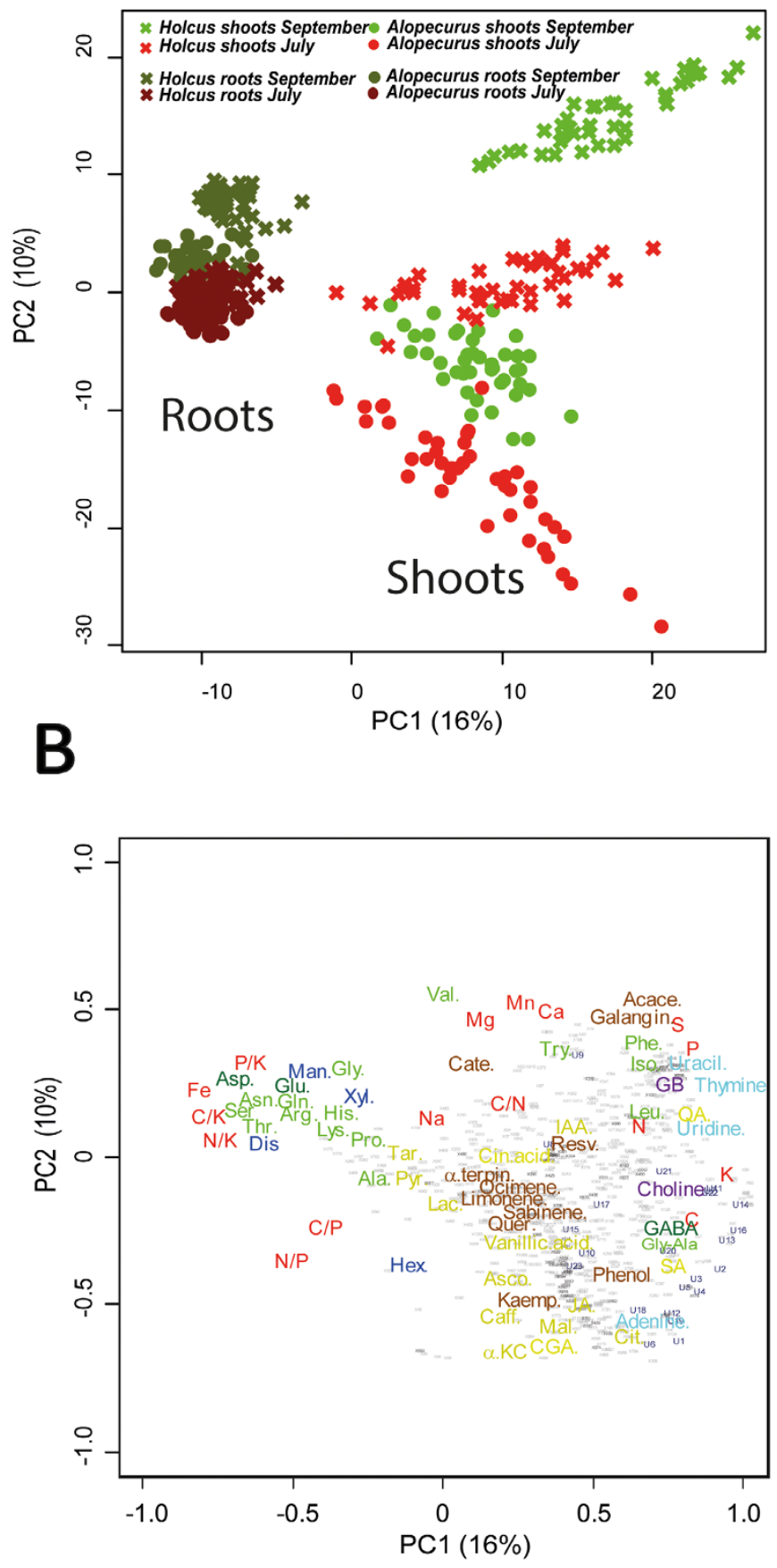

Figure $1 \mid$ Plots of cases and variables in the PCA conducted with the elemental, stoichiometric and metabolomic variables in Holcus lanatus and Alopecurus pratensis using PC1 versus PC2. (A) The cases are categorised by season and organ. Seasons are indicated by different colours (green, September; red, July). The two species are indicated by geometric figures (circles, A. pratensis; crosses, H. lanatus). Dark green and red colours are for roots, and light green and red colours are for shoots. (B) Loadings of the various elemental stoichiometric and metabolomic variables in PC1 and PC2. C, $\mathrm{N}, \mathrm{P}$, and $\mathrm{K}$ concentrations and ratios and $\mathrm{Fe}, \mathrm{Mn}, \mathrm{Mg}, \mathrm{Ca}$, and $\mathrm{S}$ concentrations are shown in red. The various metabolomic families are represented by colours: dark blue, sugars; green, amino acids; dark green, amino-acid derivatives; yellow, realted compounds to the metabolism of amino acids and sugars; cyan, nucleotides; and brown, terpenes and phenolics. Metabolites: glycine-alanine (Gly-Ala), valine (Val), tryptophan (Try), threonine (Thr), serine (Ser), lysine (Lys), leucine (Leu), proline (Pro), phenylalanine (Phe), histidine (His), glycine (Gly), glutamine (Gln), asparagine (Asn), isoleucine (Ile), arginine (Arg), alanine (Ala), glutamic acid (Glu), aspartic acid (Asp), gamma-aminobutyric acid (GABA), glycine betaine (GB), choline (Choline), tartaric acid (Tar), pyruvate (Pyr), malic acid (Mal), jasmonic acid (JA), indol acetic acid (IAA), caffeic acid (Caff), ascorbic acid (Asco), vanillic acid (Vanillic.acid), citric acid (Cit), $\alpha$-ketoglutaric acid $(\alpha \mathrm{KC})$, lactic acid (Lac), shikimic acid (SA), quinic acid (QA), chlorogenic acid (CGA), chinic acid (Cin.acid;), xylose (Xyl), hexoses (Hex), mannose (Man), disaccharide (Dis), adenine (Adenine), uracil (Uracil), thymine (Thymine), uridine (Uridine), acacetin (Acace), catechin (Cate), $\alpha$-terpinene ( $\alpha$ Terpin,), sabinene (Sabinene), resveratrol (Resv), quercetin (Quer), ocimene (Ocimene), limonene (Limonene), galangin (Galangin), kaempferol (Kaemp), phenolic group (Phenol). Unassigned metabolites are represented by small grey points. 


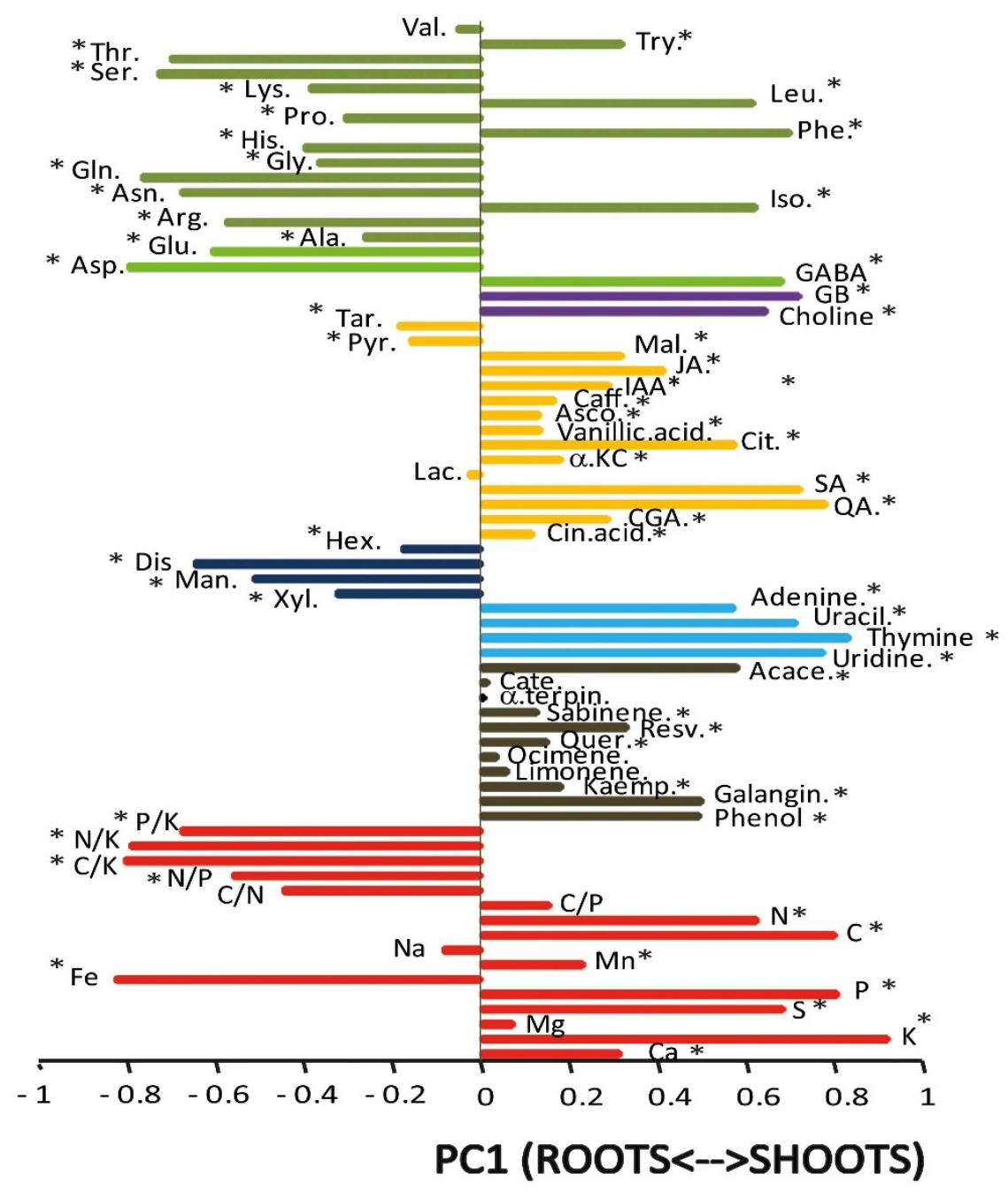

Figure $2 \mid$ Loading of elemental stoichiometric and metabolomic variables in PC1 separating shoots and roots (Fig. 1). Variables are coloured and labelled as described in the caption for Fig. 1. Asterisks showed statistical significance $(\mathrm{P}<0.05)$ in one-way ANOVAs.

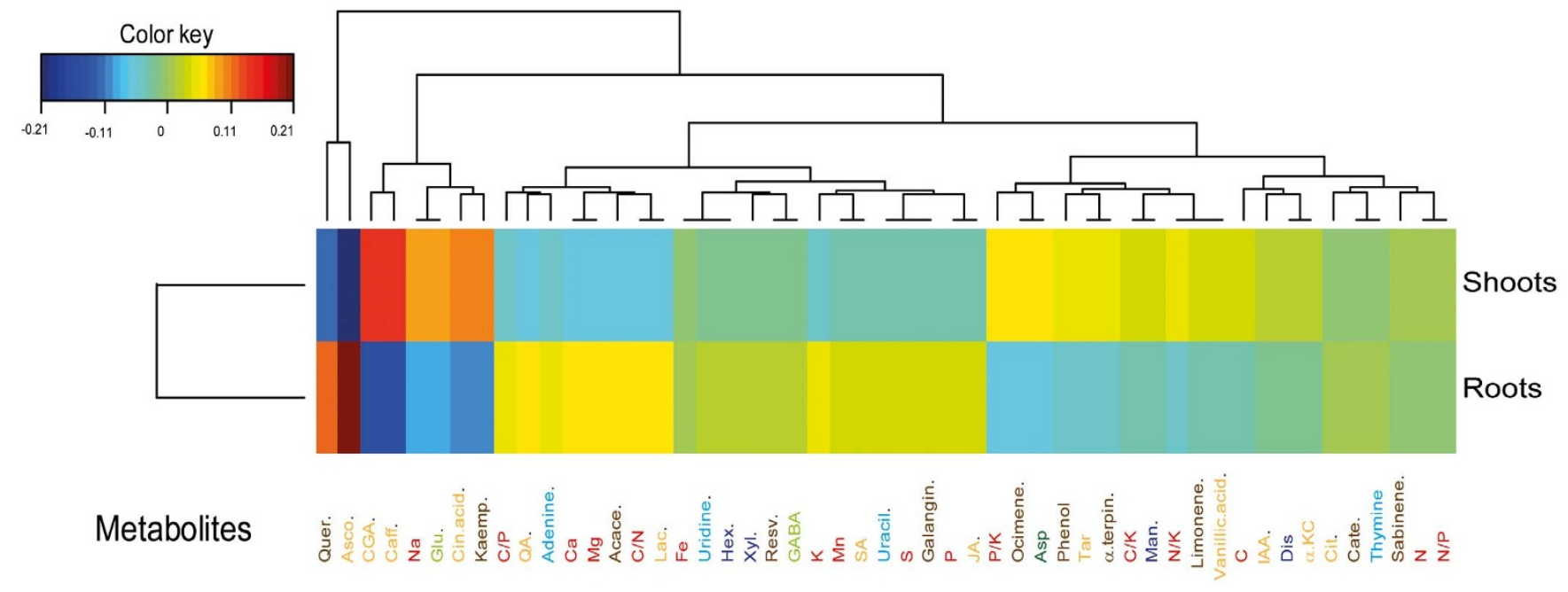

Figure $3 \mid$ Clustered image maps of the metabolites in roots and shoots based on the data of the PLS analysis. The red and blue colours indicate positive and negative correlations respectively. 


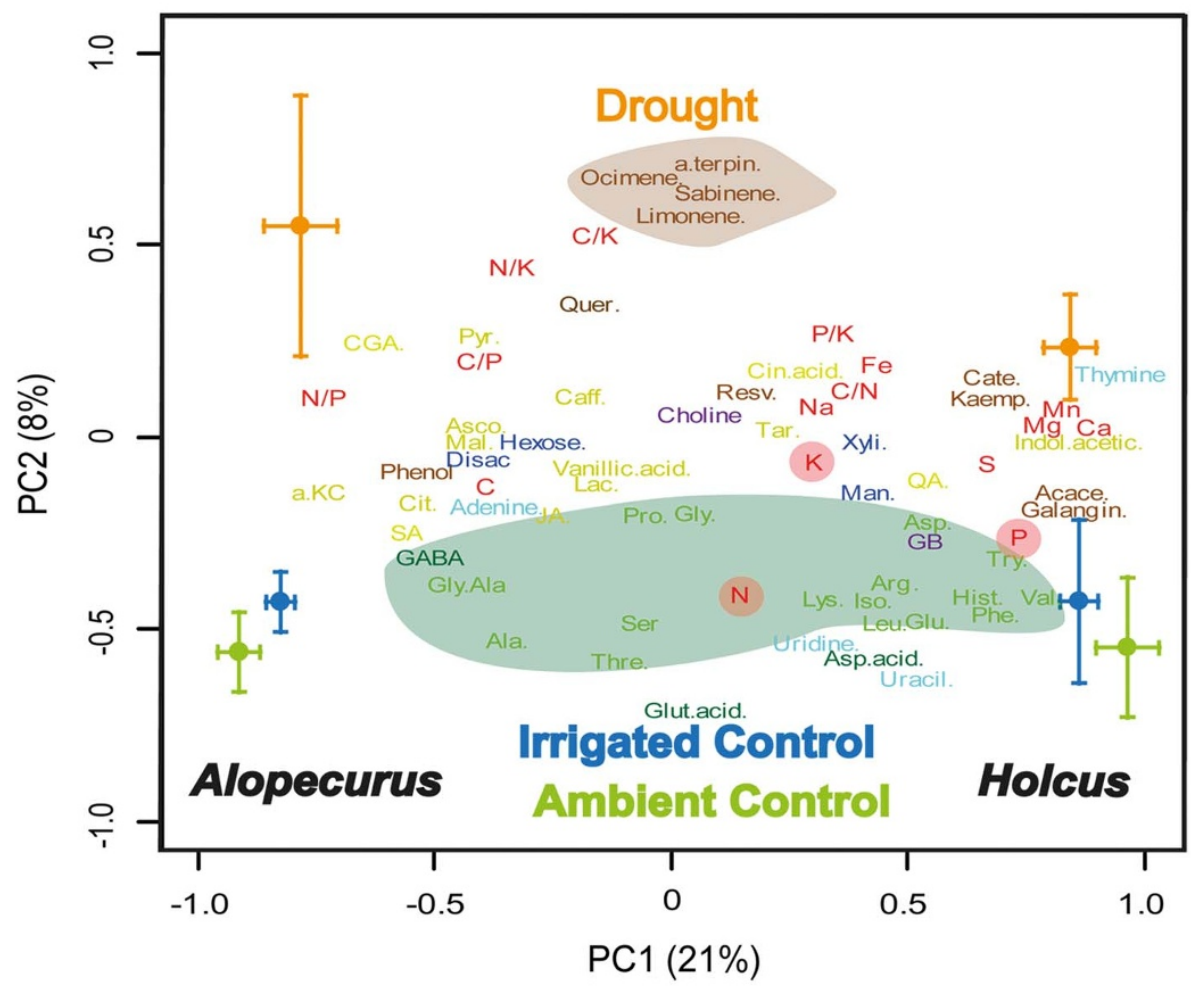

B

ROOTS

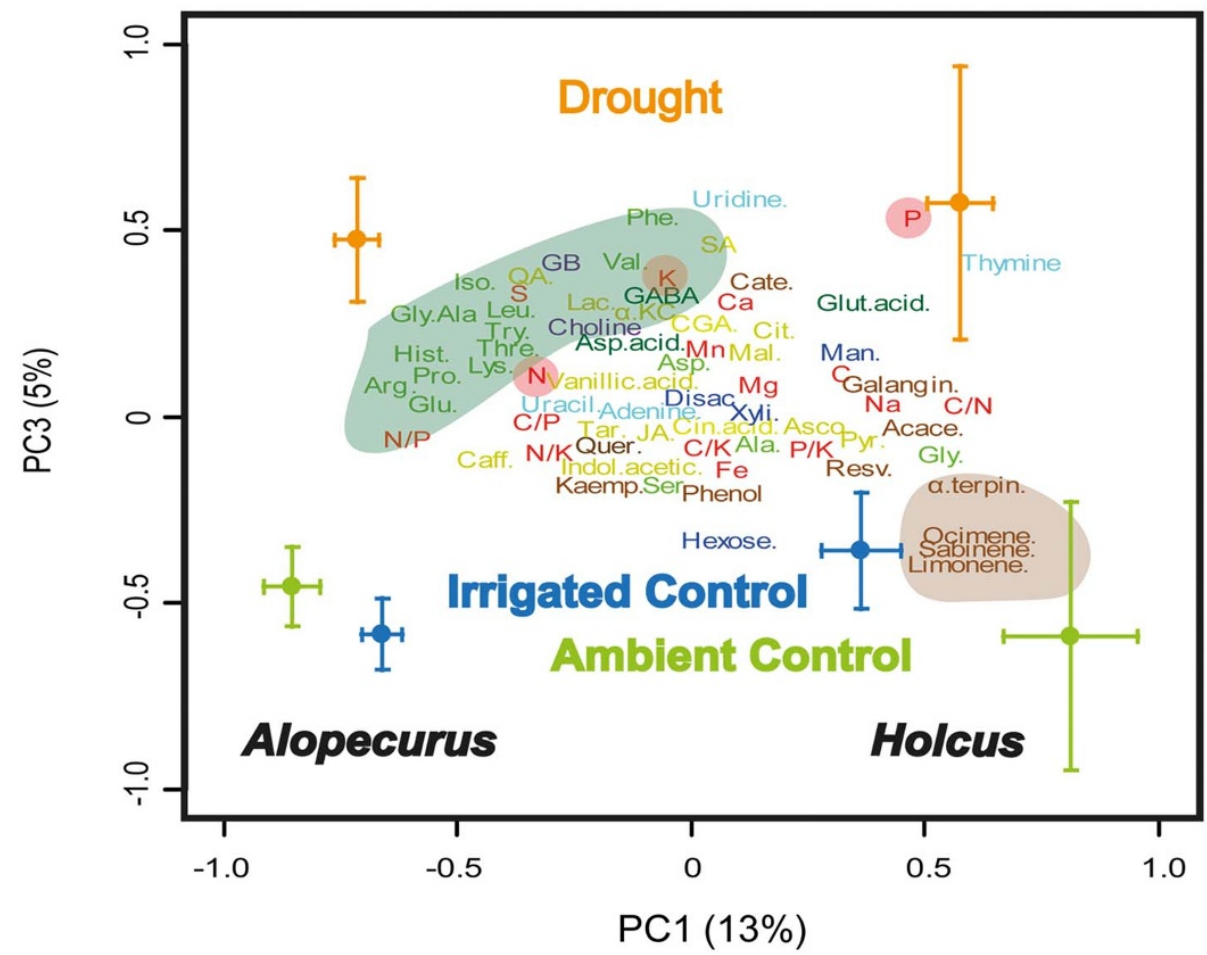

Figure $4 \mid$ Plots of cases and variables in the PCAs conducted with the elemental, stoichiometric, and metabolomic variables in plants sampled in September. (A) Plot of cases and variables for shoots. (B) Plot of cases and variables for roots. $\mathrm{C} / \mathrm{N} / \mathrm{P} / \mathrm{K}$ ratios are shown in red. The various metabolomic families are represented by colours: blue, sugars; green, amino acids; dark green, amino-acid derivatives; yellow, related compounds to the amino acids and sugars metabolism; cyan, nucleotides; violet, osmolytes; and brown, terpenes and phenols. Variables are coloured and labelled as described in the caption for Fig. 1. The means of the cases are indicated by colour: blue, irrigated control; green, ambient control; and orange, drought. Holcus lanatus is indicated as Holcus and Alopecurus pratensis as Alopecurus. 


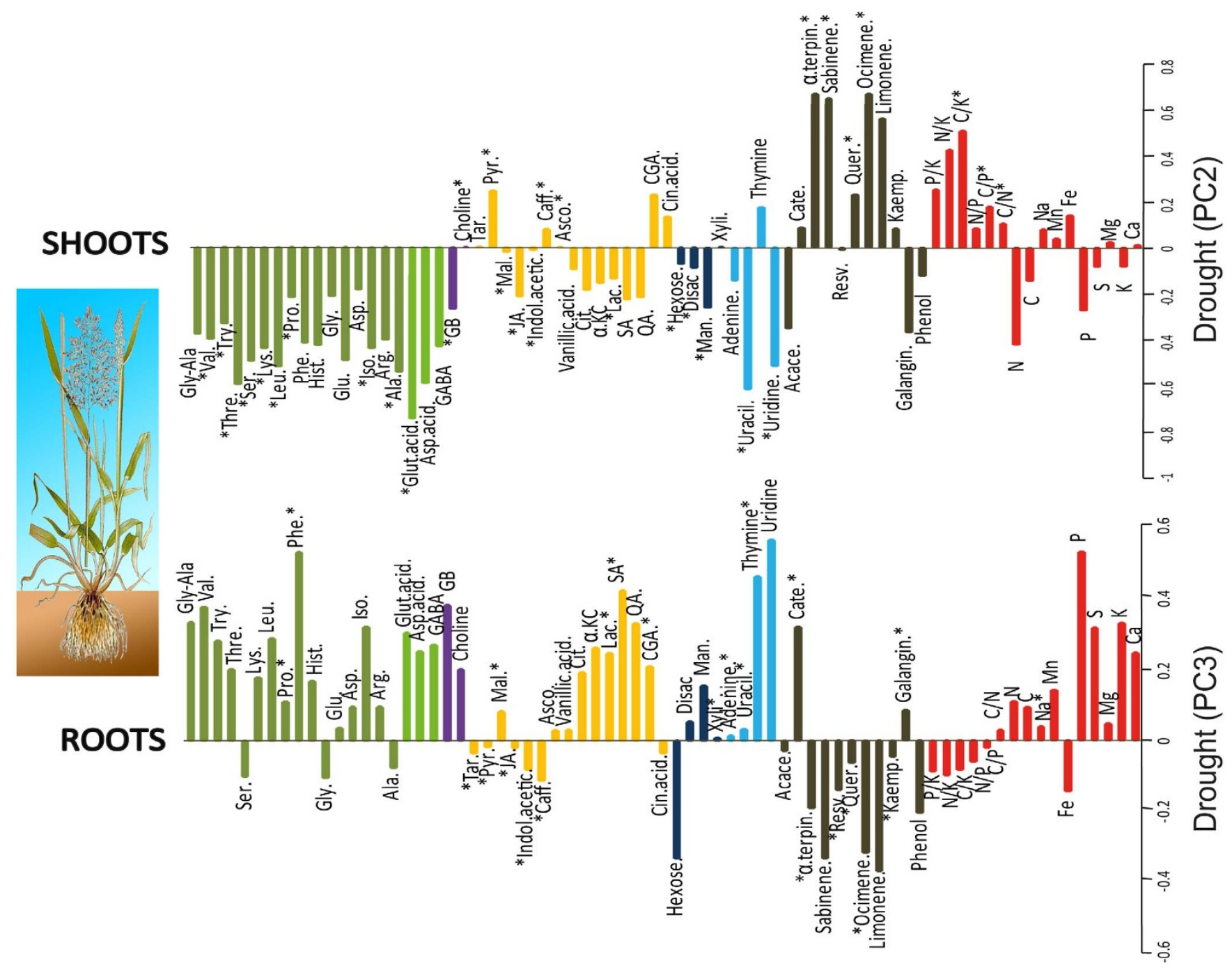

Figure 5 Loadings of elemental stoichiometric and metabolomic variables in the PC axes separating drought treatments (Fig. 3). Variables are coloured and labelled as described in the caption for Fig. 1. Asterisks showed statistical significance $(\mathrm{P}<0.05)$ in one-way ANOVAs. The drawing is a reproduction of the painting by C. A. M. Lindman.

$0.00 \mathrm{ppm})$. All handling of liquid samples, automation, and acquisition were controlled using TopSpin 3.1 software (BrukerBiospin, Rheinstetten, Germany).

Statistical analyses. The changes in the stoichiometries and metabolomes of $H$. lanatus and A. pratensis with the factors studied (shoots and roots, season, species, and drought treatment) were analysed by principal component analysis (PCA) and PERMANOVA with Euclidean distances. The PCAs were performed with the pca function of the mixOmics package of $\mathrm{R}$ (R Development Core Team 2008). The PERMANOVA as well as PLS (partial least squares) analysis, and CIM (clustered image maps) conducted with R (R Development Core Team 2008). A KolmogorovSmirnov (KS) test was performed on each variable to test for normality. All assigned and identified metabolites were normally distributed, and any unidentified metabolomic variable that was not normally distributed was removed from the data set. Statistica v8.0 (StatSoft) was used to perform the ANOVAs, post-hoc tests, and KS tests.

1. Hibberd, J. M. \& Quick, W. P. Characteristics of C4 photosynthesis in stems and petioles of C3 flowering plants. Nature 415, 451-4 (2002).

2. Brouwer, R. Distribution of dry matter in the plant. Neth J Agric Sci 10, 361-376 (1962).

3. Zerihun, A., McKenzie, B. \& Morton, J. Photosynthate costs associated with the utilization of different nitrogen - forms: influence on the carbon balance of plants and shoot - root biomass partitioning. New Phytol. 138, 1-11 (1998).

4. Penuelas, J., Biel, C. \& Estiarte, M. Chages in biomass, chlorophyll content and gas-exchange of beans and peppers under nitrogen and water-stress. Photosynthetica 29, 535-542 (1993).
5. Sims, L., Pastor, J., Lee, T. \& Dewey, B. Nitrogen, phosphorus and light effects on growth and allocation of biomass and nutrients in wild rice. Oecologia 170, 65-76 (2012).

6. Mao, W. et al. Life history strategy influences biomass allocation in response to limiting nutrients and water in an arid system. Polish J. Ecol. 60, 545-557 (2012).

7. Graf, A., Schlereth, A., Stitt, M. \& Smith, A. M. Circadian control of carbohydrate availability for growth in Arabidopsis plants at night. Proc. Natl. Acad. Sci. U. S. A. 107, 9458-9463 (2010).

8. Agren, G. I. \& Franklin, O. Root: shoot ratios, optimization and nitrogen productivity. Ann. Bot. 92, 795-800 (2003).

9. Poorter, H. \& Nagel, O. The role of biomass allocation in the growth response of plants to different levels of light, $\mathrm{CO} 2$, nutrients and water: a quantitative review. Aust. J. Plant Physiol. 27, 595-607 (2000).

10. Berendse, F. \& Möller, F. Effects of competition on root-shoot allocation in Plantago lanceolata L.: adaptive plasticity or ontogenetic drift? Plant Ecol. 201, 567-573 (2008).

11. Albaugh, T. J., Allen, H. L. \& Kress, L. W. Root and stem partitioning of Pinus taeda. Trees Struct. Funct. 20, 176-185 (2005).

12. Wilson, J. B. Shoot Competition and Root Competition. J. Appl. Ecol. 25, 279-296 (1988).

13. Agren, G. I. \& J, F. W. Modelling carbon allocation- a review. NZJ For. Sci 23 , 343-353 (1993)

14. Cannell, M. G. R. \& Dewar, R. C. Carbon Allocation in Trees: a Review of Concepts for Modelling. Adv. Ecol. Res. 25, 59-104 (1994).

15. Sardans, J., Peñuelas, J. \& Rivas-Ubach, A. Ecological metabolomics: overview of current developments and future challenges. Chemoecology 21, 191-225 (2011). 
16. Peñuelas, J. \& Sardans, J. Ecological metabolomics. Chem. Ecol. 25, 305-309 (2009).

17. Shulaev, V., Cortes, D., Miller, G. \& Mittler, R. Metabolomics for plant stress response. Physiol. Plant. 132, 199-208 (2008).

18. Carrow, R. N. Drought Resistance Aspects of Turfgrasses in the Southeast: RootShoot Responses. Crop Sci. 36, 687-694 (1996).

19. Lloret, F., Casanovas, C. \& Peñuelas, J. Seedling survival of Mediterranean shrubland species in relation to root: shoot ratio, seed size and water and nitrogen use. Funct. Ecol. 210-216 (1999).

20. Trenberth, K. E., Dai, A., Rasmussen, R. M. \& Parsons, D. B. The changing character of precipitation. Bull. Am. Meteorol. Soc. 1161, 1205-1217 (2003).

21. IPCC [Solomon, S. et al. (eds.)] [996] Climate change 2007: the physical science basis. Contribution of working group I to the fourth assessment report of the Intergovernmental Panel on Climate Change (Cambridge University Press, 2007)

22. Beniston, M. et al. Future extreme events in European climate: an exploration of regional climate model projections. Clim. Change 81, 71-95 (2007).

23. Li, F. L., Bao, W. K. \& Wu, N. Effects of water stress on growth, dry matter allocation and water-use efficiency of a leguminous species, Sophora davidii. Agrofor. Syst. 77, 193-201 (2009).

24. Wang, X. et al. Spring temperature change and its implication in the change of vegetation growth in North America from 1982 to 2006. Proc. Natl. Acad. Sci. U. S. A. 108, 1240-5 (2011).

25. Rivas-Ubach, A., Sardans, J., Pérez-Trujillo, M., Estiarte, M. \& Peñuelas, J. Strong relationship between elemental stoichiometry and metabolome in plants. Proc. Natl. Acad. Sci. U. S. A. 109, 4181-6 (2012)

26. Nunes-Nesi, A., Fernie, A. R. \& Stitt, M. Metabolic and signaling aspects underpinning the regulation of plant carbon nitrogen interactions. Mol. Plant 3 973-96 (2010)

27. Keys, A. J. et al. Photorespiratory nitrogen cycle. Nature 275, 741-743 (1978).

28. McNeil, S. D., Nuccio, M. L., Ziemak, M. J. \& Hanson, a D. Enhanced synthesis of choline and glycine betaine in transgenic tobacco plants that overexpress phosphoethanolamine N-methyltransferase. Proc. Natl. Acad. Sci. U. S. A. 98, 10001-5 (2001).

29. Kinnersleya, A. M. \& Turanob, F. J. Gamma Aminobutyric Acid (GABA) and Plant Responses to Stress. CRC. Crit. Rev. Plant Sci. 19, 479-509 (2010).

30. Álvarez, S. \& Sánchez-Blanco, M. J. Changes in growth rate, root morphology and water use efficiency of potted Callistemon citrinus plants in response to different levels of water deficit. Sci. Hortic. (Amsterdam). 156, 54-62 (2013).

31. Huang, B. \& Gaob, H. Root Physiological Characteristics Associated with Drought Resistance in Tall Fescue Cultivars. Crop Sci. 40, 196-203 (2000).

32. Peñuelas, J. \& Estiarte, M. Can elevated $\mathrm{CO}(2)$ affect secondary metabolism and ecosystem function? Trends Ecol. Evol. 13, 20-4 (1998).

33. Jentsch, A. et al. Climate extremes initiate ecosystem-regulating functions while maintaining productivity. J. Ecol. 99, 689-702 (2011).

34. Peñuelas, J. \& Staudt, M. BVOCs and global change. Trends Plant Sci. 15, 133-44 (2010)
35. Sterner, R. W., Elser, J. J. \& Hendrixson, H. Seston stoichiometry at the regional scale: What is the mean and variance of C:N:P of the average lake? Ecol. Soc. Am. Annu. Meet. 87, 50 (2002).

36. Peñuelas, J. et al. Response of plant species richness and primary productivity in shrublands along a north-south gradient in Europe to seven years of experimental warming and drought: reductions in primary productivity in the heat and drought year of 2003. Glob. Chang. Biol. 13, 2563-2581 (2007).

37. Walter, J. et al. Combined effects of multifactor climate change and land-use on decomposition in temperate grassland. Soil Biol. Biochem. 60, 10-18 (2013).

38. Rivas-Ubach, A. et al. Ecometabolomics: optimized NMR-based method. Methods Ecol. Evol. 4, 464-473 (2013).

\section{Acknowledgments}

This research was supported by the Spanish Government grants CGL2013-48074-P and CTQ2012-32436, the Catalan Government grant SGR 2014-274, the European Research Council Synergy grant ERC-2013-SyG-610028 IMBALANCE-P, the Academy of Sciences of the Czech Republic grant M200871201, the Bavarian Ministry of Sciences (FORKAST) and the German Science Foundation DFG (JE 282/61)

\section{Author contributions}

A.G.G., J.P., J.S., M.P.T., A.J., C.B. and J.K. designed the research with the help of all the other co-authors. All authors participated in the chemical and statistical analyses. A.G.G wrote the first draft and thereafter all authors, J.P., J.S., M.P.T., T.P., A.R.U., O.U., M.O., K.V., A.J., J.K. and C.B. contributed to the writing of the manuscript and the drafting of the figures.

\section{Additional information}

Supplementary information accompanies this paper at http://www.nature.com/ scientificreports

Competing financial interests: The authors declare no competing financial interests.

How to cite this article: Gargallo-Garriga, A. et al. Opposite metabolic responses of shoots and roots to drought. Sci. Rep. 4, 6829; DOI:10.1038/srep06829 (2014).

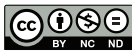

This work is licensed under a Creative Commons Attribution-NonCommercialNoDerivs 4.0 International License. The images or other third party material in this article are included in the article's Creative Commons license, unless indicated otherwise in the credit line; if the material is not included under the Creative Commons license, users will need to obtain permission from the license holder in order to reproduce the material. To view a copy of this license, visit http:// creativecommons.org/licenses/by-nc-nd/4.0/ 